\title{
La valeur aspectuelle - problème de traduction (Confrontation franco-tchèque)
}

\author{
Miroslava Sládková \\ Université Charles de Prague, \\ Faculté des Lettres, Institut de Traductologie
}

The aspect quality - a translation problem (French-Czech confrontation) This article is a reflection on the problem of verbal aspect in Czech and in French and on related translation problems. Czech verbs have a binary opposition of perfectivity versus imperfectivity; in French the situation is more complex, where the nature of the verbal action (Aktionsart) usually comes into play. In Slavonic languages aspect and temporality features differ; in French the temporal information prevails. The problem arises when the translator must respond to the relevant aspect opposition in Czech. It would be incorrect to try to introduce the categories of one language into another, typologically distant, language. Usually, both studied languages behave rather differently in this sphere and the problem of aspect must therefore be resolved at text level.

\section{Introduction}

Les théories de plusieurs études linguistiques concernant l'aspect verbal diffèrent considérablement.

Pour certains, tout ce qui n'est pas identique au fonctionnement du verbe slave, qui a suscité le terme même d'aspect, n'est pas aspect. [...] Pour d'autres, à l'extrémité opposée, toute valeur apparemment introduite par le verbe, si elle ne relève pas du 'temps' ou du 'mode', est à caractériser comme aspective. (Cohen:7)

Les théories slaves et francophones diffèrent en effet sur de nombreux points

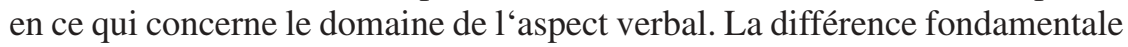
entre les deux systèmes est évidente et, par conséquent, elle engendre des difficultés dans le domaine de la traduction. Avant de confronter le français et le tchèque à partir des problèmes liés à l'aspectualité, essayons d'abord de caractériser l'aspect verbal slave en prenant l'exemple du tchèque.

\section{L'aspect verbal}

\subsection{Situation en tchèque}

Le système verbal tchèque conçoit l'action soit "comme globale (perfective) ou, au contraire, non globale (imperfective: action conçue comme étant en cours ou se développant)[...]" (Šabršula 1986:219-220). Il s'agit d'une 
opposition grammaticale mais qui influence également le sens du verbe. C'est une catégorie qui relève à la fois du lexique et de la grammaire. "Le système slave présente cette originalité d'opposer, pour l'expression de l'aspect, des verbes complets différents et non pas des conjugaisons complémentaires dans un même verbe." (Cohen:30) L'aspectualité est donnée au verbe a priori, elle y est présente tout le temps, (même à l'infinitif), ce qui est impensable, par exemple, en français. En plus de cette opposition binaire fondamentale, certains auteurs distinguent une troisième forme aspectuelle, le fréquentatif, qui exprime l'itération - l'action dans sa répétition. C'est une forme non actuelle qui, dans la traduction vers une langue étrangère, peut être traduite soit par le même verbe que les formes précédentes soit par un verbe itératif. En effet, l'itération est ici lexicalement si nette qu'on peut la traduire même dans une langue qui désigne l'aspectualité d'une façon entièrement différente.

Ainsi, par exemple, les formes infinitives tchèques zpívat (imperf.) - zazpívat (perf.) ne peuvent être traduites en français que par une seule forme - chanter; la troisième forme - le fréquentatif (zpívávat) - peut même être traduite à l'infinitif (avoir l'habitude de chanter, aimer chanter, arriver à quelqu'un de chanter). Cet exemple ne se limite évidemment pas au français; ainsi psávat = zu schreiben pflegen, scribere solere, to be used to write. Il est intéressant de noter que le tchèque ne connaît pas de forme marquée actuelle, mais emploie une forme marquée non actuelle (psávám = j'ai l'habitude d'écrire, déávám = j'ai l'habitude de faire quelque chose). Dans d'autres langues, il existe en revanche des formes marquées qui servent à exprimer l'action actuelle comme, en anglais, I am writing, en italien, sto scrivendo, en français, je suis en train d'écrire; des formes non marquées existent également: I write, scrivo ou j'écris. (D'après Kope ný:6)

Comme nous l'avons déjà dit, en tchèque, la majorité des verbes coexistent dans des couples aspectuels, perfectif - imperfectif. Mais il existe quelques verbes dont la sémantique exclut soit la perfectivité, soit l'imperfectivité. Si c'est la perfectivité qui est exclue, on parle de verbes imperfectiva tantum. Nous pouvons citer à titre d'exemple les verbes modaux (muset 'devoir', moci 'pouvoir', chtit 'vouloir') et quelques verbes

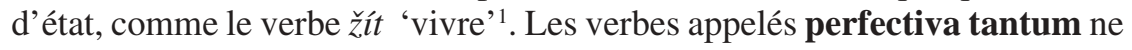
peuvent pas, par contre, exprimer l'imperfectivité; c'est le cas par exemple de verbes comme nadchnout se 's'enthousiasmer', prospat (dopoledne) 'passer la matinée à dormir', 'faire la grasse matinée'. En outre, il existe en tchèque une série de verbes d'origine étrangère comme absorbovat 'absorber', izolovat 'isoler', organizovat 'organiser'. Ces verbes sont appelés verbes à double aspect. Le contexte détermine s'ils sont perfectifs ou imperfectifs. Quelques-uns sont néanmoins capables de créer des formes perfectives par préfixation (organizovat - zorganizovat 'organiser'). (D'après P íru ní mluvnice e šiny)

En regard des soi-disant catégories objectives (comme celle, par exemple, du temps verbal), on peut se poser la question de l'objectivité ou de la subjectivité de l'aspect verbal. Le Dictionnaire de linguistique (Dubois et al.:53) apporte la définition suivante: 
L'aspect est une catégorie grammaticale qui exprime la représentation que se fait le sujet parlant du procès exprimé par le verbe (ou par le nom d'action), [...] alors que les temps, les modaux et les auxiliaires de temps expriment les caractères propres du procès indiqué par le verbe indépendamment de cette représentation du procès par le sujet parlant. (cité par Cohen:16)

L'aspect est donc considéré dans cette définition comme une catégorie subjective. Les grammairiens allemands qui, au XIX ${ }^{\mathrm{e}}$ siècle, ont introduit le concept d'Aktionsart (= mode d'action, ordre du procès) l'ont défini comme objectif par opposition à l'aspect proprement dit qui serait subjectif (D'après Cohen:34). Dans ce contexte, on cite souvent l'exemple de Vilém Mathesius, l'un des représentants du Cercle linguistique de Prague, qui, à partir de deux phrases qui diffèrent uniquement du point de vue de l'aspect verbal employé, en démontre la subjectivité:

(1)

Pamatuji se, že jsem ti tu knihupjil (perf.). = Je me souviens de t'avoir prêté ce livre.

Pamatuji se, že jsem ti tu knihu p joval (imperf.). = Je te l'ai pourtant prêté, ce livre, je m'en souviens.

(D’après Mathesius:196)

Dans la première phrase (avec l'aspect perfectif - pjil ), la même réalité extralinguistique est conçue tout simplement comme un fait s'étant déroulé au passé, tandis que dans la deuxième phrase (l'aspect imperfectif p joval ), cette même réalité est envisagée comme s'il s'agissait d'une scène qui se passe sous nos yeux. La réalité reste la même, mais l'aspect imperfectif comprend un certain "quantum dubitatif": un peu comme si le locuteur essayait de se persuader lui-même d'avoir vraiment prêté ce livre, de ne pas s'être trompé.

Cependant, dans d'autres cas, l'aspect ne peut pas être considéré comme subjectif; l'exemple du schéma d'incidence suivant le démontre:

Kdyžpsal dopis, zazvonil telefon. (imperf.) Il écrivait (était en train d'écrire) une lettre, quand le téléphone a sonné. Alors qu'il écrivait (était en train d'écrire) une lettre, le téléphone a sonné.

Když napsal dopis, zazvonil telefon. (perf.) Quand il a eu fini d'écrire une lettre, le téléphone a sonné. (Alors qu'il avait fini d'écrire..., Après qu'il ait eu fini d'écrire...)

(D’après Hamplová:6)

Dans l'exemple cité, l'aspect reflète d'une façon objective la réalité donnée. On peut donc constater que l'aspect verbal soit apprécie et exprime une action 
réelle d'une manière subjective, soit se rapporte à des actions objectivement données.

\subsection{Situation en français}

Le français n'a pas de moyens morphologiques pour les oppositions aspectuelles marquées dans les langues slaves. Il emploie d'autres moyens linguistiques, tels que des "désignants lexématiques (racines, préfixes, suffixes, verbes et leurs catégories morphologiques, sémions verbaux complexes, formations prémorphologiques, adverbes, [...]) qui se combinent de façon raffinée avec les moyens syntaxiques." (Šabršula 1990:85). Les linguistes slaves font la différence entre l'aspect et l'ordre du procès (mode d'action, mode de procès, Aktionsart), tandis que les théories françaises confondent souvent ces notions. La valeur aspectuelle des verbes français doit être envisagée, plus qu'en tchèque, dans un contexte défini. Assez souvent, c'est l'information temporelle qu'il faut prendre en considération. ${ }^{2}$ Il est également difficile de qualifier les verbes a priori, sans prendre en considération leur complément éventuel.

L'exemple suivant démontre l'ambivalence des participes passés dont l'aspectualité se constitue en fonction des compléments respectifs dans le texte:

\section{un journal lu $\quad x \quad$ un journal lu par tout le monde une leçon répétée $x$ une leçon répétée chaque jour Les portes sont fermées $x$ Les portes sont fermées tous les jours à 10 heures du soir.}

(Référovskaïa-Vassiliéva:292)

La différence entre les verbes téliques et atéliques considérée par quelques linguistes comme une différence aspectuelle ne résout pas non plus le problème. Par définition, les verbes téliques se distinguent par un trait sémantique particulier: une fois commencé, le procès conduit nécessairement à un terme qui en constitue l'achèvement. Arriver, mourir, tuer, vaincre sont ainsi des verbes téliques. En revanche, les verbes atéliques (jouer, parler, travailler, aimer) ne comprennent pas ce trait sémantique. La plupart des verbes français atéliques correspondent aux verbes tchèques imperfectifs; cependant, il existe des verbes français atéliques ayant deux équivalents en tchèque, l'un imperfectif et l'autre perfectif, ainsi 'jouer' = hrát (si), zahrát (si). Les verbes français téliques correspondent presque toujours en tchèque aux deux aspects, par exemple 'arriver' = $p$ ijit, $p$ icházet, 'tuer' $=$ zabit , zabíjet. ${ }^{3}$ Les équivalents tchèques des verbes tels 'naître' ou 'mourir' peuvent exister soit dans leur forme perfective, soit imperfective. ('naître' = narodit $s e$, rodit se, 'mourir' = zem ít, umírat ). Citons, à ce propos, la fameuse scène reproduisant la naissance d'un éphémère dans la pièce des frères apek $D e$ la vie des insectes où l'aspect imperfectif du verbe naître est pertinent et, par conséquent, doit être traduit en français. 
(6)

Celá zem se chv je!

$N$ co velkého se d je!

Já se rodim!

(K. apek, J. apek:284)

Toute la terre tremble.

Quelque chose de grand se prépare.

Moi, je nais.

(Frères apek:130)

De même qu'en tchèque, l'équivalent français exprime dans ce dernier exemple un processus. D'ailleurs, un peu plus loin, pour la même situation, le traducteur a employé une tournure encore plus expressive, à savoir: Je suis en train de naître. (107) Dans cette perspective, certaines affirmations de linguistes ${ }^{4}$ apparaissent plus qu'étonnantes. Ainsi la définition de Wagner et Pinchon citée par Monnerie-Goarin (13):

Les procès signifiés par les verbes perfectifs comportent par eux-mêmes, indépendamment de tout effet extérieur exercé sur eux, une limitation. Une fois commencé, le procès va nécessairement à un terme qui en constitue l'achèvement. Naître et mourir sont perfectifs: on ne peut pas continuer à naître ou mourir dès le moment où l'on est né ou mort.

L'opposition télique vs atélique ne peut donc pas représenter l'oppostion aspectuelle. Les slavisants ne la comprennent même pas comme relevant de l'ordre du procès proprement dit. On pourrait la définir comme une catégorie qui se trouve à cheval entre l'aspect et l'ordre du procès. Néanmoins, en français, cette opposition joue un rôle important dans le système des temps du passé, en ayant une influence sur la sémantique du verbe. Les verbes atéliques sont aussi susceptibles dans certains contextes d'aboutir à un terme défini. Prenons l'exemple de l'opposition écrire vs écrire un livre. Certains linguistes - parmi eux Sten et Garey - considèrent que le verbe écrire, employé dans une phrase du type: Hier, j'ai écrit une lettre, est un exemple de l'aspect perfectif. (En ce qui concerne le tchèque, il y a deux équivalents possibles - perfectif et imperfectif.) Mais il faut néanmoins tenir compte dans cet exemple de la sémantique: il suffit de remplacer le complément d'objet une lettre par un autre, comme par exemple ma thèse, et l'aspect verbal change. Il en est de même pour des modifications telles que:

Hier, j'ai écrit ma thèse vs L'année dernière, j'ai écrit ma thèse.

Cependant, ces interprétations aspectuelles dépendent du contexte et, même ici, l'appréhension de la seconde phrase peut être équivoque. (D'après Hamplová:11-14)

A ce propos, il s'avère utile d'employer le terme de valeur aspectuelle qui représente la signification aspectuelle que revêt l'énoncé tout entier. ${ }^{5}$ 


\subsection{Quelques problèmes de traduction}

Prenons maintenant un problème spécifique, à savoir l'aspectualité des temps du passé, plus précisément l'opposition "aspectuelle" passé composé (passé simple) vs imparfait. Assez souvent, les linguistes tendent à identifier l'aspect perfectif à "l'aspect" limitatif et vice versa. (Monnerie-Goarin: 19). Dans les pays francophones, maintes théories concernant l'aspect verbal désignent l'opposition passé simple (passé composé) vs imparfait d'opposition aspectuelle. Pour illustrer cette affirmation, citons l'extrait d'un chapitre des Cahiers Chronos:

Rappelons que l'existence d'éventuels imparfaits perfectifs ou passés simples imperfectifs ne peut pas servir d'argument contre le contenu conceptuel aspectuel de l' imparfait et du passé simple. [...] En examinant les critiques de la théorie aspectuelle, on constate que les contre-exemples traditonnels vont toujours dans le même sens: la perfectivité du passé simple n'est jamais contestée; (Vetters-De Mulder:26)

Voyons à ce propos quelques exemples tirés des textes français traduits en tchèque et vice versa. Les exemples suivants démontrent que l'opposition binaire perfectivité vs imperfectivité du verbe tchèque ne correspond pas toujours à la conception de l'aspectualité en français. Dans les exemples (7) et (8), l'équivalent tchèque du passé simple français est le verbe imperfectif. L'équivalent du passé composé de l' exemple (9) est également un verbe imperfectif tchèque:

\section{(7)}

Maigret parvenait vraiment à avoir l'air maussade.

Un crime crapuleux? grommela-t-il.

Certainement pas....

Lecoeur fouilla parmi ses paperasses.

La victime s'appelle Hélène Lange. Elle avait quarante-huit ans ...

(Maigret à Vichy:37)

Maigretovi se opravdu poda ilo tvá it se nep ív tiv.

“Je to loupežná vražda?” zabru el.

"Ur it ne."

$\boldsymbol{P}$ ehraboval se vapírech. (Lecoeur)

“Ob t' se jmenuje Helena Langeová. Bylo jí ty icet osm let ...

(Maigret v lázních:28)

... m li ocho eného králíka, kterýs nimi žil, na krok se od nich nehnul $\cdots$

(Nesmrtelnost:101)

... avaient apprivoisé un lapin qui vécut ensuite avec eux sans les quitter d'un pas...

(L'immortalité:146) 
(9)

Nous avons vérifié au Carlton, où elle a bien joué au bridge jusqu'à onze heures vingt.

(Maigret à Vichy:71)

Ov ili jsme si v Carltonu, že tam opravdu hrála bridž až do jedenácti dvaceti.

(Maigret v lázních:54)

L'exemple (10) prouve que l'aspect verbal tchèque est susceptible de distinguer de très fines nuances sémantiques dont la compréhension est souvent un grand problème pour un étranger. L'équivalent textuel du verbe français conseiller est le verbe tchèque doporu ovat 'recommander'. Sa variante imperfective, employée dans notre texte, et le contexte dans lequel elle s'inscrit impliquent que la personne n'a pas obéi aux conseils de son interlocuteur, tandis que $1^{\prime}$ aspect perfectif, en regard du contexte approprié, exprimerait plutôt le contraire:

Faut-il que je vous rappelle les phrases de votre interlocuteur? Ne vous a-t-il pas conseillé, justement, de rester encore deux ou trois jours à Vichy?

(Maigret à Vichy:132)

Je t eba, abych vám p ipomn l, co íkal volající? Nedoporu oval vám práv on, abyste z stala ješt dva t i dny ve Vichy?

(Maigret v lázních:101)

Le "paradoxe imperfectif" représente un autre phénomène intéressant. D'après Vetters-De Mulder:30, “[...] l'imparfait n'a pas encore perdu sa valeur aspectuelle, tout en admettant que l'affaiblissement de son imperfectivité, qui devra finalement mener à la neutralisation de sa valeur aspectuelle, a bien commencé."

L'imparfait narratif (de rupture, pittoresque, dynamique ou impressionniste) dont les effets de sens sont parfois décrits comme accélération ou décélération (Bres:67) se comporte dans certains cas comme le prétérit allemand. Pour ce qui est de la traduction en tchèque, cet emploi exige assez souvent l'aspect perfectif (11) et (12) ou, éventuellement, le présent historique (13):

Le chasseur frappait à une porte. Une voix répondait “entrez”. On lui ouvrait le battant et Maigret se trouvait dans un salon.

(Maigret voyage:111)

Poslí ek zaklepal na dve e. Ozvalo se "dále”. P ed Maigretem se otev elo k ídlo dve í, ocitl se v salonu. 
(12)

“To, co mi íkáte, m nezajímá!" ... a v té chvíli p iložila prsty obou rukou k hrudi. ... Pak zaklonila mírn hlavu, tvá pokryla úsm vem a paže hodila prudce, a p ece ladn, dop edu.

(Nesmrtelnost:163)

“Ce que vous dites là ne m’intéresse pas.” [...] répondait-elle le bout des doigts sur sa poitrine. [...] Puis elle rejetait légèrement la tête en arrière et, le visage voilé d'un sourire, elle lançait brusquement, mais avec élégance, ses bras en avant.

(L’immortalité:197)

Leur petite comtesse appelait le médecin, geignait, se laissait transporter à l'hôpital puis, en douce, téléphonait, d'abord à Paris, cherchant à rejoindre son premier mari ...

(Maigret voyage:105)

Povedená komteska; nejd ív si s úp ním zavolá pro doktora, nechá se odvézt do nemocnice, pak obtelefonuje Pa íz, aby sehnala svého prvního manžela...

(Maigret na cestách:63)

Le dernier exemple (14) concerne l'imparfait qui exprime l'itération. L'équivalent tchèque de l'imparfait français est un verbe perfectif, parce que ce qui compte, c'est l'achèvement de l'action. L'itération est suffisamment exprimée lexicalement.

(14)

Cela faisait simplement partie de leur routine de passer à ce moment près du kiosque, où il n'y avait d'ailleurs pas de la musique tous les jours.

Certains soirs, cette partie du parc était presque déserte. Ils la traversaient, tournaient à droite, s'engageaient dans l'allée couverte qui longeait une rue pleine d'enseignes lumineuses.

(Maigret à Vichy:12)

Zvykli si jen prost projít v této chvíli kolem pavilónu, kde ostatn nebyla hudba každý den.

N které ve ery bývala tato ást parku tém liduprázdná. Prošli jí, zahnuli doprava a dali se klenutou alejí, která vedla soub žn s ulicí plnou neón .

(Maigret v lázních:9)

\section{Conclusion}

Dans le présent article, nous avons étudié la valeur aspectuelle au travers de la confrontation du tchèque et du français. Le tchèque, en tant que 
langue slave, connaît l'opposition binaire perfectivité vs imperfectivité et les marques d'aspect diffèrent des marques temporelles; les marques aspectuelles sont attachées à des formes verbales qu'elles soient conjuguées ou non. Par contre, en français, c'est l'information temporelle qui prévaut. La situation y est beaucoup plus complexe et, parfois, la catégorie d'ordre du procès entre en jeu. L'opposition imparfait vs passé simple (passé composé) ne représente pas automatiquement une opposition aspectuelle, comme le démontre le choix de nos exemples.

Le problème se pose pour le traducteur lorsqu'il doit réagir à une opposition aspectuelle pertinente en tchèque. Il est impossible de chercher à importer dans une langue les catégories d'une autre, surtout lorsque cellesci sont typologiquement éloignées. Dans ce domaine, les deux langues se comportent d'une façon différente et c'est souvent au niveau textuel qu'il faut résoudre les problèmes relevant de l'aspectualité.

\section{Bibliographie}

\section{Oeuvres d'où sont extraits les exemples:}

apek, Karel \& Josef apek (1982). Ze spole né tvorby. Praha: eskoslovenský spisovatel, Praha.

Frères apek (1978). De la vie des insectes. Traduit par M.Tomášková. Praha: Dilia.

Kundera, Milan (1993). Nesmrtelnost. Brno: Atlantis.

Kundera, Milan (1990). L'Immortalité. Traduit par E. Bloch. Paris: Gallimard.

Simenon, Georges (1971). Maigret à Vichy. Paris: Presses de la Cité.

Simenon, Georges (1970). Maigret v lázních. Traduit par E. Drmolová. Praha: Spirála.

Simenon, Georges (1958). Maigret voyage. Paris: Presses de la Cité.

Simenon, Georges (1989). Maigret na cestách. Traduit par L. Blana ová. Praha: Melantrich.

\section{Ouvrages théoriques:}

Bres, Jacques (2000). "Un emploi discursif qui ne manque pas de style: l'imparfait en cotexte narratif." Anne Carlier, Véronique Lagae \& Céline Benninger (éd.) (2000). Passé et parfait. Amsterdam/Atlanta GA: Editions Rodopi, 59-77.

Cohen, David (1989). L'aspect verbal. Paris: Presses universitaires de France.

Confais, Jean-Paul (1995). Temps, mode, aspect. Toulouse: Presses Universitaires du Mirail.

Dubois, Jean et al. (1973). Dictionnaire de linguistique. Paris: Larousse.

Garey, Howard B. (1957). "Verbal Aspect in French". Language 33, No 2, 91-110.

Garnier, Georges (1986). "L'aspect verbal en anglais et en français. Note de systématique comparée.” Michel Ballard (1986). La traduction. De la théorie à la didactique. Presses Universitaires de Lille, 79-98.

Guillemin-Flescher, Jacqueline (1981). Syntaxe comparée du français et de l'anglais. Paris: Éditions Ophrys. 
Hamplová, Sylva (1994). K problematice vidovosti vitalštin. Praha: Univerzita Karlova.

Karlík, Petr, Nekula, Marek \& Zde ka Rus nová (éd.) (1995). P íru ní mluvnice eštiny. Praha: Lidové noviny.

Kope ný, František (1962). Slovesný vid v eštin. Praha: s. Akademie v d.

Kuszmider, Barbara (1999). Aspect, temporalité et modalité en polonais et en français. Linguistique contrastive et traduction, $\mathrm{N}^{\circ}$ spécial, Paris: Éditions Ophrys.

Mathesius, Vilém (1947). eština a obecný jazykozpyt. Praha: Melantrich.

Monnerie-Goarin, Annie (1996). Les temps du passé et l'aspect du verbe. Paris: Didier/Hatier.

Référovskaïa, Elizaveta Arturovna \& Alla Konstantinovna Vassiliéva (1973). Essai de grammaire française. Leningrad: Prosveš enie.

Sládková, Miroslava (1998). "Etude contrastive de l'aspect en tchèque et en français dans une perspective textuelle." Acta Universitatis Palackianae Olomucensis, Facultas Philosophica, Philologica 71, 185-190.

Sládková, Miroslava (2001). "L'aspect verbal en tant que problème de traduction." Linguistica Pragensia, Vol. XI/1, 29-38.

Sten, Holger (1952). Les temps du verbe fini (indicatif) en français moderne. Copenhague: Ejnar Munksgaard..

Šabršula, Jan (1962). Nomináln verbální konstrukce a povaha d je ve francouzštin . Praha: Univerzita Karlova

Šabršula, Jan (1986). Problèmes de la stylistique comparée française-tchèque et tchèque-française. Praha: SPN.

Šabršula, Jan (1990). Problèmes de la stylistique comparée du français et du tchèque. AUC, Monographia CIV Praha: UK.

Vetters, Carl \& Walter De Mulder (2000). "Passé simple et imparfait: contenus conceptuel et procédural." Anne Carlier, Véronique Lagae \& Céline Benninger (éd.) (2000). Passé et parfait. Amsterdam/Atlanta GA, 13-36.

\footnotetext{
${ }^{1}$ Dans ce contexte, il est très intéressant de mentionner que le système verbal français permet d'effectuer une différence entre: j'ai dîu-je devais, j'ai pu-je pouvais, j'ai voulu - je voulais, il a fallu - il fallait. Il s'agit d'une différence sémantique dont le tchèque n'est pas capable; il doit exprimer celle-ci autrement, par exemple en se référant au contexte.

2 "En français les procès actualisés dans le temps du récit comportent souvent de faibles marques aspectuelles." (Guillemin-Flescher:105)

${ }^{3}$ Il est vrai que la différence se manifeste quelquefois aussi dans la construction passive (l'ennemi est vaincu x il est aimé de tous), mais pas toujours. Dans ce contexte, j'aimerais rappeler la possibilité qui existe en italien - la différence entre: il nemico è battuto $x$ il nemico viene battuto. Pour ce qui est de la traduction en tchèque, cette fois-ci il s'agit d'une véritable différence aspectuelle.

${ }^{4}$ voir par exemple Confais:210.

${ }^{5}$ voir Kuszmider.
} 